\title{
High Pressure Freezing Airway Smooth Muscle Tissue at Physiological Length for Analysis of Contractile Filaments
}

\author{
Tera L. Lavoie ${ }^{1}$, Maria L. Dowell ${ }^{2}$, Jotham R. Austin II ${ }^{1,3}$, Julian Solway ${ }^{4}$ \\ 1. University of Chicago, Office of Shared Research Facilities. Chicago, IL, USA \\ 2. University of Chicago, Department of Pediatrics. Chicago, IL, USA \\ 3. University of Chicago, Department of Molecular Genetics and Cell Biology. Chicago, IL, USA \\ 4. University of Chicago, Department of Medicine. Chicago, IL, USA
}

The ability of smooth muscle to shorten to a greater extent than other muscle types is one key feature for smooth muscle function in many organs. Although much progress has been made in understanding smooth muscle function [1,2], our knowledge about the smooth muscle myofilament network at the ultrastructural level is much less developed. Understanding of contraction in airway smooth muscle (ASM) has been based on the sliding filament theory of contraction developed for striated muscle [3]. Specifically, force generation is the result of side polar myosin filament heads pushing against the actin filament network. Dense body structures dispersed throughout the cytosol are comprised of $\alpha$-actinin [4] and are thought to be analogous to Z-disks in striated muscle [5].

Unlike in striated muscle, the exact arrangement of the contractile filaments in smooth muscle has not been elucidated, as prior imaging techniques have not been able to provide enough resolution to fully discern individual actin and myosin filaments along their entire lengths. Therefore, key data such as individual filament lengths and analysis of filament connectivity have been previously impossible to obtain accurately. Additionally, maintaining physiologic length is key when preserving smooth muscle, as the contractile filaments are labile.

In this study, we developed a method to high pressure freeze (HPF) unstimulated and isometrically contracted human tracheal smooth muscle (HTSM) strips. To maintain muscle length, we clamped the HTSM strip within the sample planchets just prior to freezing (Figure 1A). Micrographs show well preserved cells in which individual filaments can be discerned. Because of the accurate preservation of these samples and the lack of potential artifacts aldehydes and other chemical fixatives can introduce, an accurate 3-dimensional structure of the contractile filaments was created, allowing for analysis of actinmyosin-actin connectivity within the ASM. Here we show that the total content of both actin and myosin filaments appear to increase after ACh stimulation; furthermore, the total numbers of both filaments appear to increase after ACh isometric contraction.

Methods in brief: human tracheal smooth muscle (HTSM) strips were dissected from tracheas, fastened at both ends with aluminum foil clips, pinned out on a platform at approximate in vivo length, and allowed to equilibrate at their physiological length for at least 30 minutes before the beginning of the freezing protocol. Aluminum sample planchets were secured, well side up, to the freezing apparatus with silicon grease. The open freezing apparatus was then slid under the tissue and tightly clamped around the tissue, securing the tissue at the equilibrated, physiologic length within the aluminum planchets (Figure 1A). HTSM strips were frozen in a Baltec HPM 010 high-pressure freezer, freeze-substituted in $0.1 \%$ uranyl acetate $/ 0.25 \%$ gluteraldehyde in acetone at $-85^{\circ} \mathrm{C}$, and infiltrated with lowcryl HM-20 resin. 
Three-dimensional, hand-segmented models of actin and myosin filaments were created from tomograms of both unstimulated and isometrically contracted HTSM strips. A representative image of the unstimulated muscle cell and the corresponding filament model are shown in Figure 1B and 1C, respectively. Using the hand-segmented models, actin and myosin filament numbers, total polymerized content, and filament lengths were calculated. Both actin and myosin filament numbers appear to increase after ACh stimulation when compared to unstimulated tissues. Actin filaments show an increase by 2.3 fold and myosin filaments by 4.4 fold after ACh stimulation. Moreover, total polymerized content of both actin (2.78 fold increase) and myosin filaments (6.62 fold) appear to increase after ACh stimulation. However, there was no difference in the filament length distributions of either actin or myosin filaments comparing stimulated to unstimulated tissues [6].

\section{References:}

[1] ML Dowell et al, Eur Respir J 36 (2010), p. 630.

[2] TL Lavoie et al, Am J Respir Crit Care Med 186 (2012), p. 225.

[3] J Hanson and HE Huxley, Nature 172 (1953), p. 530.

[4] JV Small and JM Squire, J Mol Bio 67 (1972), p. 117.

[5] CL Prosser, Annu Rev Physil 36 (1974), p. 503.

[6] The tissue for this study was provided by Gift of Hope Organ and Tissue Donor Network through the generous gift of donor families.
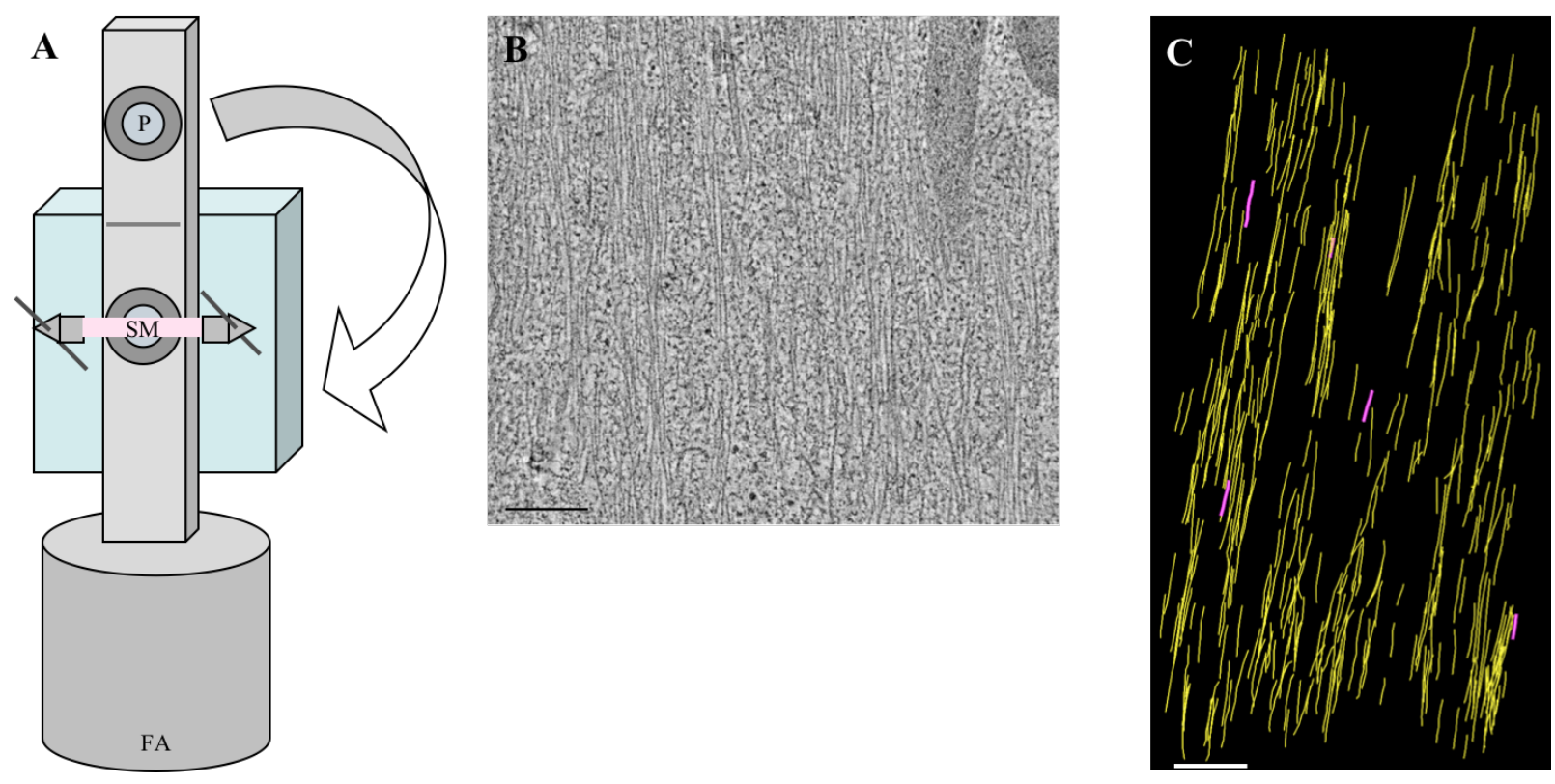

Figure 1. A. Schematic showing how human tracheal smooth muscle strips were prepared for high pressure freezing. $\mathrm{P}=$ Planchet; $\mathrm{SM}=$ Smooth Muscle Strip; FA = Freezing Apparatus. B. Longitudinal view of unstimulated HTSM cell. Thin and thick myofilaments course through the cell along the longitudinal axis. Bar $=200 \mathrm{~nm}$. C. Model of unstimulated HTSM strip. Actin filaments are yellow; myosin filaments are pink. $\mathrm{Bar}=200 \mathrm{~nm}$. 